\title{
SUPPLEMENTARY MATERIALS: TESTING SIGNIFICANCE OF FEATURES BY LASSOED PRINCIPAL COMPONENTS
}

\author{
By Daniela M. Witten, Robert Tibshirani
}

\section{1. $R$ code for simulations.}

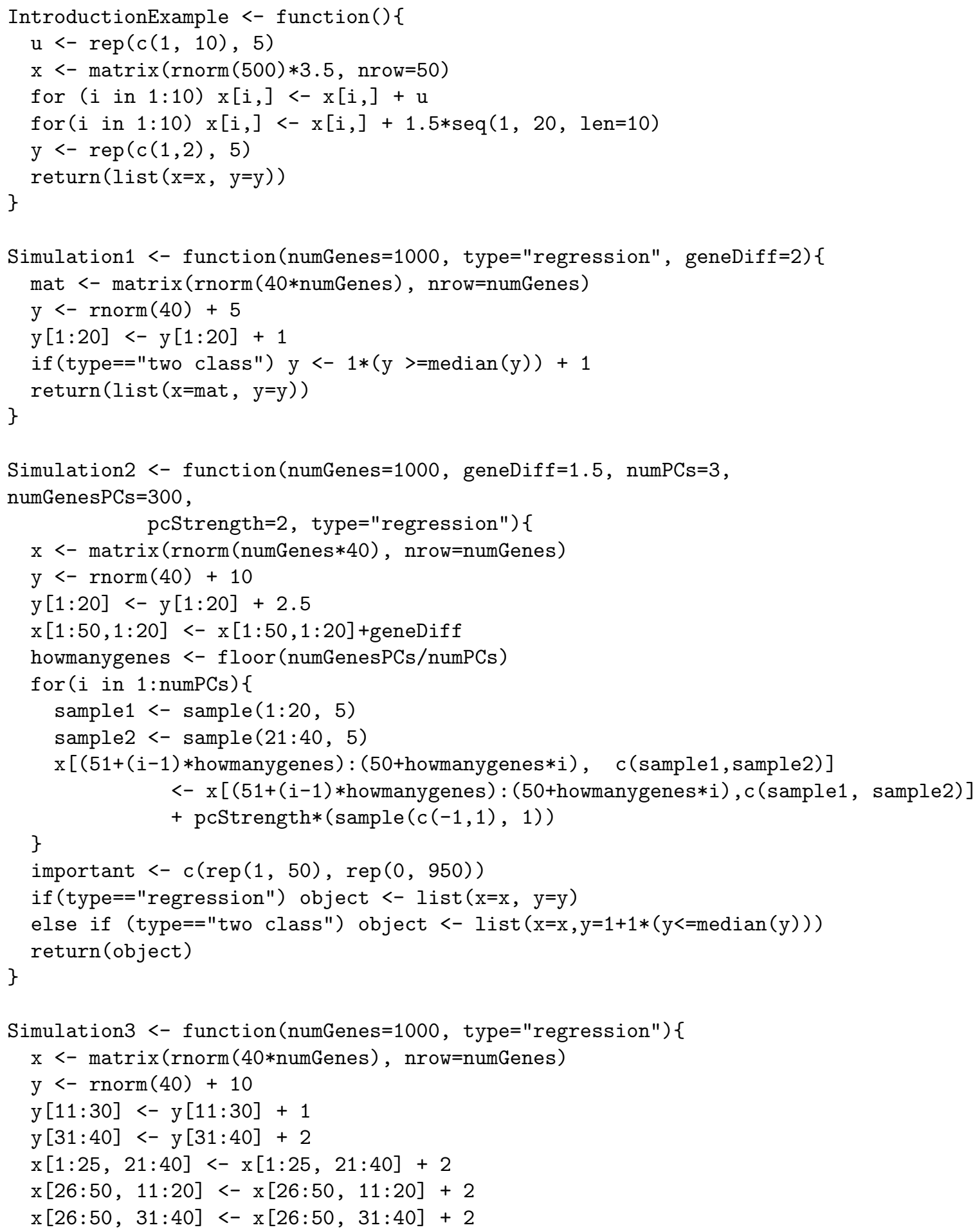




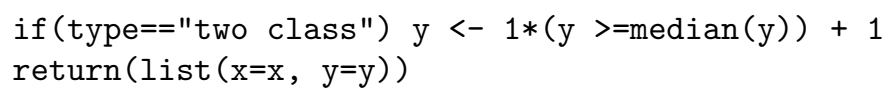

2. Details of variance derivations for latent variable model. Here, we provide details for the calculations of $\operatorname{Var}(\mathbf{T}), \operatorname{Var}\left(T_{j}\right)$, and $\operatorname{Var}\left(\hat{T}_{j}\right)$, given in Appendix D.

$$
\begin{aligned}
\operatorname{Var}\left(\mathbf{X}_{j}^{T} \mathbf{y}\right) & =\operatorname{Var}\left(\left(c_{1} \alpha_{1 j} \mathbf{u}_{1}+c_{2} \alpha_{2 j} \mathbf{u}_{2}+\mathbf{z}_{j}\right)^{T}\left(\beta_{1} \mathbf{u}_{1}+\epsilon\right)\right) \\
& =\operatorname{Var}\left(c_{1} \alpha_{1 j} \beta_{1}+\beta_{1} \mathbf{u}_{1}^{T} \mathbf{z}_{j}+c_{1} \alpha_{1 j} \mathbf{u}_{1}^{T} \epsilon+c_{2} \alpha_{2 j} \mathbf{u}_{2}^{T} \epsilon+\mathbf{z}_{j}^{T} \epsilon\right) \\
& =\operatorname{Var}\left(\beta_{1} \mathbf{u}_{1}^{T} \mathbf{z}_{j}+c_{1} \alpha_{1 j} \mathbf{u}_{1}^{T} \epsilon+c_{2} \alpha_{2 j} \mathbf{u}_{2}^{T} \epsilon+\mathbf{z}_{j}^{T} \epsilon\right) \\
& =\operatorname{Var}\left(\beta_{1} \mathbf{u}_{1}^{T} \mathbf{z}_{j}\right)+\operatorname{Var}\left(\left(c_{1} \alpha_{1 j} \mathbf{u}_{1}+c_{2} \alpha_{2 j} \mathbf{u}_{2}\right)^{T} \epsilon\right)+\operatorname{Var}\left(\mathbf{z}_{j}^{T} \epsilon\right) \\
& +2 \operatorname{Cov}\left(\beta_{1} \mathbf{u}_{1}^{T} \mathbf{z}_{j}, \mathbf{z}_{j}^{T} \epsilon\right)+2 \operatorname{Cov}\left(\left(c_{1} \alpha_{1 j} \mathbf{u}_{1}+c_{2} \alpha_{2 j} \mathbf{u}_{2}\right)^{T} \epsilon, \mathbf{z}_{j}^{T} \epsilon\right) \\
& =\beta_{1}^{2} \operatorname{Var}\left(z_{i j}\right)+\left(c_{1}^{2} \alpha_{1 j}^{2}+c_{2}^{2} \alpha_{2 j}^{2}\right) \operatorname{Var}\left(\epsilon_{i}\right)+\operatorname{Var}\left(\mathbf{z}_{j}^{T} \epsilon\right)
\end{aligned}
$$

Also, for $j \neq k$,

$$
\begin{aligned}
\operatorname{Cov}\left(\mathbf{X}_{j}^{T} \mathbf{y}, \mathbf{X}_{k}^{T} \mathbf{y}\right) & =\operatorname{Cov}\left(\left(c_{1} \alpha_{1 j} \mathbf{u}_{1}+c_{2} \alpha_{2 j} \mathbf{u}_{2}+\mathbf{z}_{j}\right)^{T}\left(\beta_{1} \mathbf{u}_{1}+\epsilon\right),\left(c_{1} \alpha_{1 k} \mathbf{u}_{1}+c_{2} \alpha_{2 k} \mathbf{u}_{2}+\mathbf{z}_{k}\right)^{T}\left(\beta_{1} \mathbf{u}_{1}+\epsilon\right)\right) \\
& =\operatorname{Cov}\left(\left(c_{1} \alpha_{1 j} \mathbf{u}_{1}+c_{2} \alpha_{2 j} \mathbf{u}_{2}\right)^{T}\left(\beta_{1} \mathbf{u}_{1}+\epsilon\right),\left(c_{1} \alpha_{1 k} \mathbf{u}_{1}+c_{2} \alpha_{2 k} \mathbf{u}_{2}\right)^{T}\left(\beta_{1} \mathbf{u}_{1}+\epsilon\right)\right) \\
& =\operatorname{Cov}\left(c_{1} \alpha_{1 j} \beta_{1}+\left(c_{1} \alpha_{1 j} \mathbf{u}_{1}^{T}+c_{2} \alpha_{2 j} \mathbf{u}_{2}^{T}\right) \epsilon, c_{1} \alpha_{1 k} \beta_{1}+\left(c_{1} \alpha_{1 k} \mathbf{u}_{1}^{T}+c_{2} \alpha_{2 k} \mathbf{u}_{2}^{T}\right) \epsilon\right) \\
& =\operatorname{Cov}\left(\left(c_{1} \alpha_{1 j} \mathbf{u}_{1}^{T}+c_{2} \alpha_{2 j} \mathbf{u}_{2}^{T}\right) \epsilon,\left(c_{1} \alpha_{1 k} \mathbf{u}_{1}^{T}+c_{2} \alpha_{2 k} \mathbf{u}_{2}^{T}\right) \epsilon\right) \\
(2.2) & =\left(c_{1}^{2} \alpha_{1 j} \alpha_{1 k}+c_{2}^{2} \alpha_{2 j} \alpha_{2 k}\right) \operatorname{Var}\left(\epsilon_{i}\right)
\end{aligned}
$$

So $\operatorname{Var}(\mathbf{T})=\operatorname{Var}\left(\mathbf{X}^{T} \mathbf{y}\right)=\left(c_{1}^{2} \alpha_{1} \alpha_{1}^{T}+c_{2}^{2} \alpha_{2} \alpha_{2}^{T}\right) \operatorname{Var}\left(\epsilon_{i}\right)+I_{p \times p}\left(\beta_{1}^{2} \operatorname{Var}\left(z_{i j}\right)+\operatorname{Var}\left(\mathbf{z}_{j}^{T} \epsilon\right)\right)$. This means that

$$
\begin{aligned}
\operatorname{Var}\left(\hat{T}_{j}\right) & =\alpha_{1 j}^{2} \alpha_{1}^{T}\left(\left(c_{1}^{2} \alpha_{1} \alpha_{1}^{T}+c_{2}^{2} \alpha_{2} \alpha_{2}^{T}\right) \operatorname{Var}\left(\epsilon_{i}\right)+I_{p \times p}\left(\beta_{1}^{2} \operatorname{Var}\left(z_{i j}\right)+\operatorname{Var}\left(\mathbf{z}_{j}^{T} \epsilon\right)\right)\right) \alpha_{1} \\
& =\alpha_{1 j}^{2} \alpha_{1}^{T}\left(\left(c_{1}^{2} \alpha_{1} \alpha_{1}^{T}+c_{2}^{2} \alpha_{2} \alpha_{2}^{T}\right)\right) \alpha_{1} \operatorname{Var}\left(\epsilon_{i}\right)+\alpha_{1 j}^{2} \alpha_{1}^{T} \alpha_{1}\left(\beta_{1}^{2} \operatorname{Var}\left(z_{i j}\right)+\operatorname{Var}\left(\mathbf{z}_{j}^{T} \epsilon\right)\right) \\
& =\alpha_{1 j}^{2} c_{1}^{2} \operatorname{Var}\left(\epsilon_{i}\right)+\alpha_{1 j}^{2}\left(\beta_{1}^{2} \operatorname{Var}\left(z_{i j}\right)+\operatorname{Var}\left(\mathbf{z}_{j}^{T} \epsilon\right)\right)
\end{aligned}
$$

From equation 2.1 above,

$$
\operatorname{Var}\left(T_{j}\right)=\operatorname{Var}\left(\mathbf{X}_{j}^{T} \mathbf{y}\right)=\left(c_{1}^{2} \alpha_{1 j}^{2}+c_{2}^{2} \alpha_{2 j}^{2}\right) \operatorname{Var}\left(\epsilon_{i}\right)+\beta_{1}^{2} \operatorname{Var}\left(z_{i j}\right)+\operatorname{Var}\left(\mathbf{z}_{j}^{T} \epsilon\right)
$$

Therefore, we have reduced the variance by projecting $T_{j}$ onto $\alpha_{1}$. 
3. Supporting Figures. 

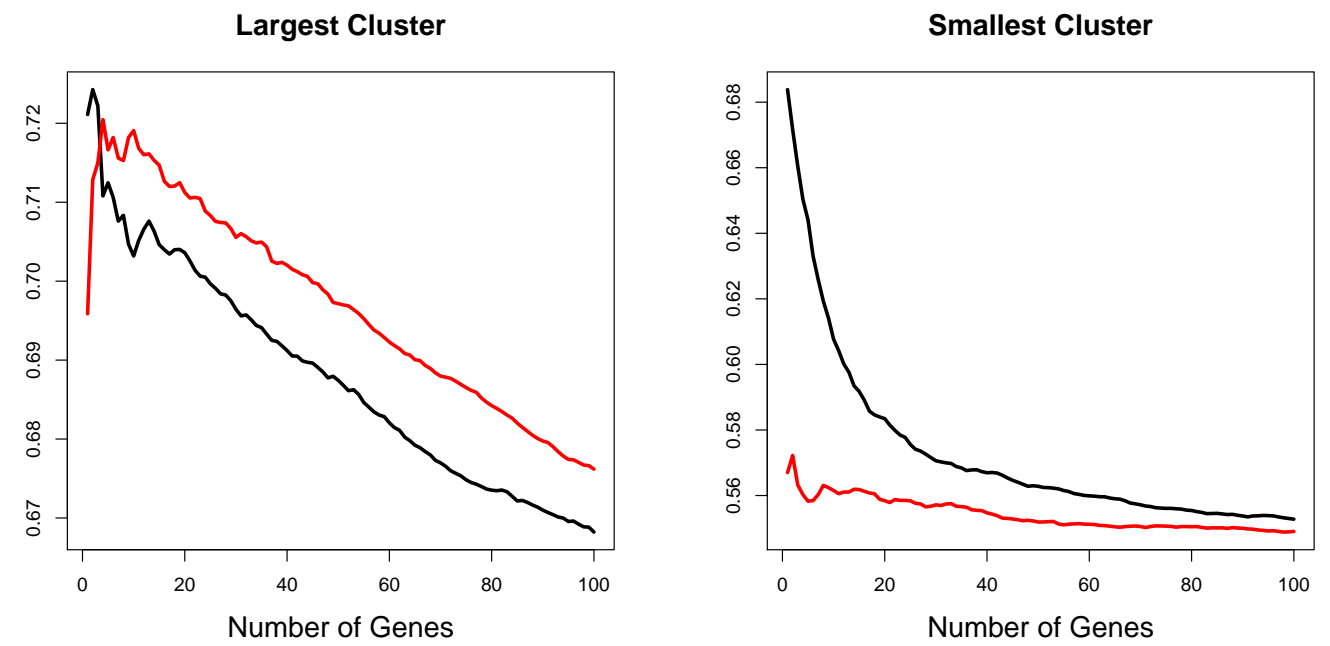

Fig 1. $\mathrm{E}\left(\left|T_{j}{ }^{*}\right||| L_{j} \mid>c_{2}(\alpha)\right)$ (red) and $\mathrm{E}\left(\left|T_{j}{ }^{*}\right||| T_{j} \mid>c_{1}(\alpha)\right.$ ) (black) are shown for two simulations based on the colon cancer $X$ data. Predictive advantage of LPC over $T$ is positive when $y$ is taken to be the centroid of a large gene cluster. It is negative when $y$ is taken to be the centroid of a small gene cluster. 

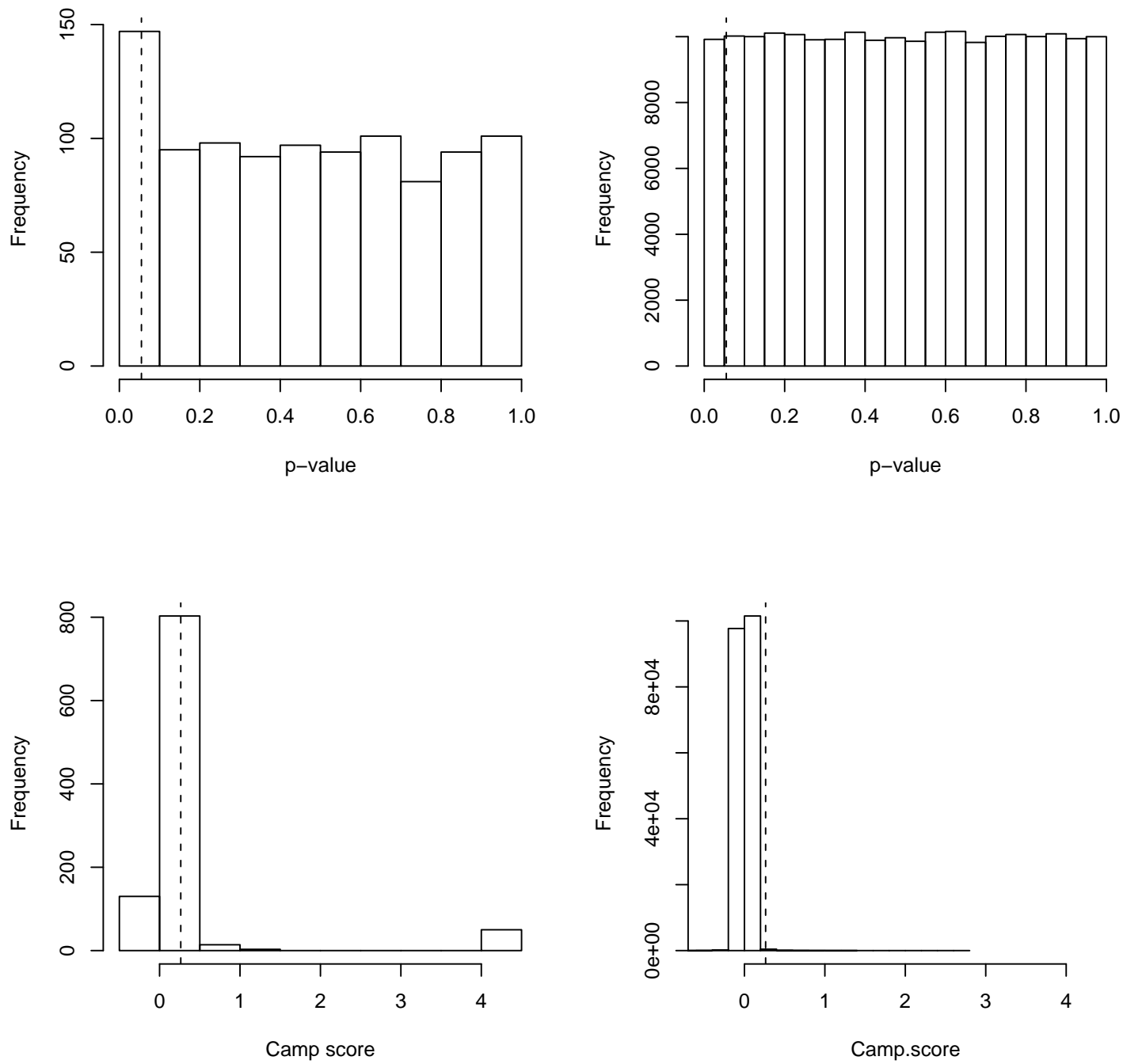

FIG 2. A simulation experiment illustrates the problem of functionally dependent statistics. The top left panel is a histogram of 1000 p-values, uniform on [0,1], except for the first 50 which equal $10^{-6}$. In the top right panel, simulated null p-values from $U[0,1]$ approximate the null p-values in the top left. These simulated null p-values can be used to estimate the FDR. However, the results for the CaMP score (bottom panels) show that the null scores in the bottom right do not provide a good approximation to the non-null (lower) scores in the bottom left. For reference, a vertical broken line is drawn at the 10\% point (top panels) and the $90 \%$ point (bottom panels). 

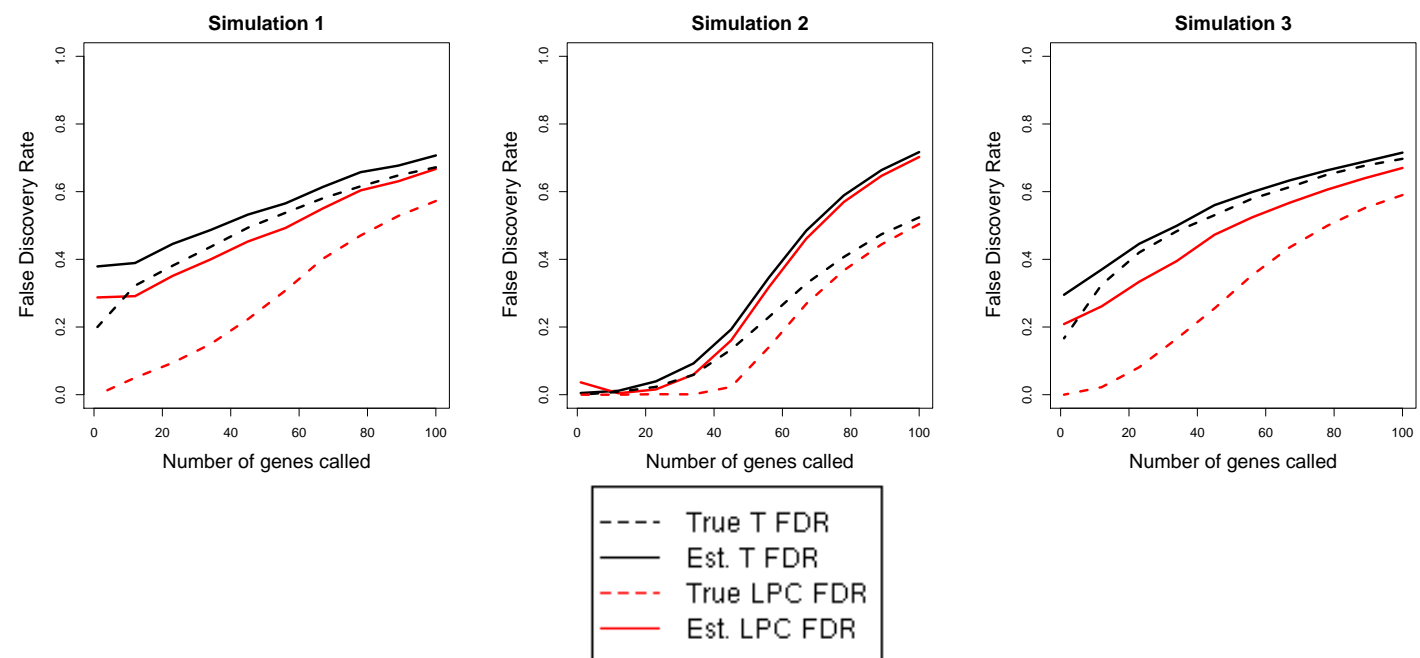

FIG 3. Estimated FDR for both $T$ and $L P C$ for Simulations 1, 2, and 3 for a quantitative response variable. The estimated LPC FDR is conservative. LPC is red, T is black. Estimated FDR is solid and true FDR is dashed.
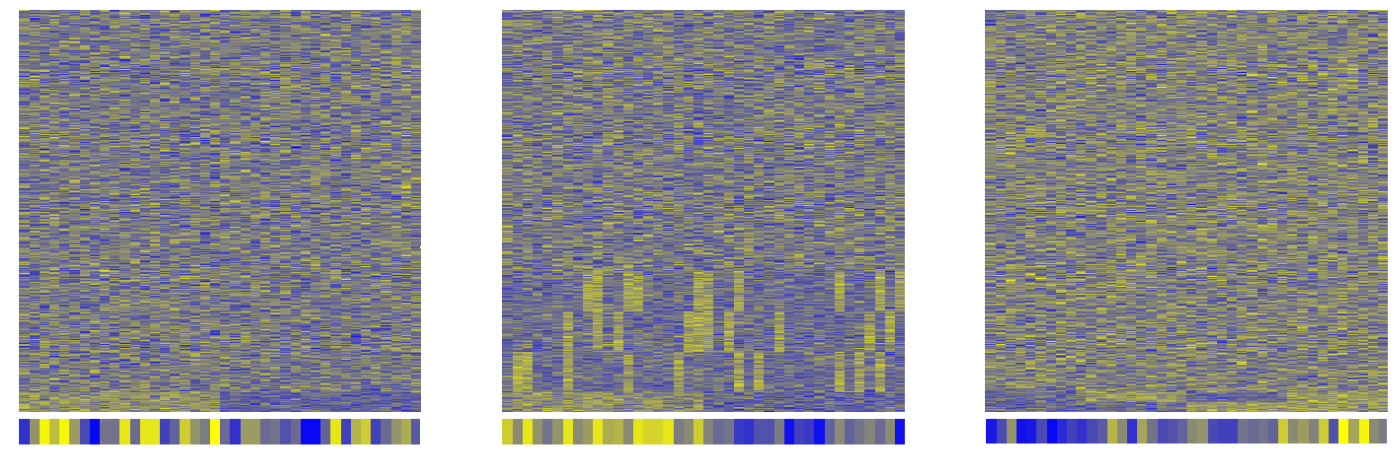

FIG 4. From left to right, heatmaps for Simulations 1, 2, and 3 are shown. The bottom panel shows the outcomes for each of the 40 observations. The bottom 50 genes on each heatmap are significant. 